\title{
Francesco Giuseppe Cabrera, il principe messicano degli umanisti di America nei secoli XX e XXI ${ }^{1}$
}

Francisco José Cabrera, el príncipe mexicano de los humanistas de América en los siglos XX y XXI

\author{
Tarsicio HERRERA ZAPIÉN \\ Centro de Estudios Clásicos, IIFL, Universidad Nacional Autónoma de México \\ tarsicioherrera@yahoo.com.mx
}

\begin{abstract}
RiASSUNTO: La piú recente Eneide creata in America nei due secoli scorsi, che é Monumenta Mexicana del avvocato Francesco Giuseppe Cabrera recentemente tramontato (marzo 2015, 99 anni), tratta come monumento in tutto culminante, le cittá, gli eroi e i poeti del Messico attraverso la sua storia. In rimarcabile parallello, mentre l'Eneide di Virgilio raduna 10,000 esametri in dodici libri narrativi del poeta; sul contrario le Monumenta Mexicana di Cabrera radunano in 4,500 esametri ed undici foglietti quasi tutta la storia e la leggenda del Messico e dei suoi personaggi. Questi undici volumetti succedono ai perdurabili poemi epico lirici del gesuiti del 1700, Abad, Landívar, Fuentes e López.
\end{abstract}

Resumen: La más reciente Eneida creada en América en los dos siglos pasados, que es Monumenta Mexicana del abogado Francisco José Cabrera, recién fallecido (marzo, 2015, 99 años), presenta, como un monumento del todo culminante, las ciudades, los héroes y los poetas de México, a través de su historia. En notable paralelo, mientras la Eneida de Virgilio agrupa 10,000 hexámetros en 12 libros narrativos del Paduano, por el contrario Cabrera agrupa en 4,500 hexámetros y once folletos casi toda la historia y la leyenda de México y de sus personajes. Estos once folletos suceden a los perdurables poemas épico-líricos de los jesuitas del 1700: Abad, Landívar, Fuentes y López.

Parole ChiAVE: America; Virgilio; Cabrera; Diego; volume; leggenda.

Palabras Clave: América; Virgilio; Cabrera; Diego; episodio; leyenda.

RECIBIDO: 13 de mayo de 2016 • ACEPTADO: 15 de agosto de 2016.

DOI: 10.19130/iifl.nt.2015.33.2.736

Sono lieto di mostrarvi quella che è la più recente Eneide neolatina creata in America nei due secoli più moderni. È il volume intitolato

\footnotetext{
${ }^{1}$ Testo presentato in italiano per l'autore stesso como conferenza magistrale nel XIV Congresso de la FIEC (Fédération Internationale des Associations d'Études Classiques) a Bordeaux, Francia, in agosto 2014.
} 
Monumenta Mexicana (la Eredità messicana) dal suo autore Francesco Giuseppe Cabrera in esametri neolatini.

Vi lo descriverò. Mentre Virgilio narra nella Eneide originale la leggenda dei guerrieri troiani in via di fondare la città di Roma e fa che Enea s'incontri con delle dee, delle dame e dei guerrieri, anche nel suo turno, il poeta Francesco Cabrera raduna nelle sue Monumenta Mexica$n a$, i momenti culminanti delle città, degli eroi e dei poeti del Messico. Questa Eneide americana è un capolavoro di questo avvocato Francesco Giuseppe Cabrera, poeta nato a Puebla nel 1916, 98 anni fa ¡ed oggi recentemente tramontato!

Poi viene un parallelo rimarcabile: che l'Eneide romana congrega 10,000 esametri in 12 libri conclusi dal mantovano Virgilio nel 19 a. C. Ed a sua volta, le Monumenta Mexicana di Cabrera abbracciano non 12 ma sì 11 fascicoli di episodi drammatici in quasi cinquemila esametri, $\mathrm{i}$ cui successivi protagonisti danno nome a ciascun fascicolo.

Queste Monumenta le ha create il giurista messicano Francesco Cabrera due secoli dopo la più memorabile serie di poemi eroici del suo continente. Mi riferisco ai magnifici poemi neolatini che scrivevano i gesuiti esiliati dalla Spagna e dall'America nel secolo XVIII da Carlo III. E perchè furono esiliati i gesuiti? Perché insegnavano le nazioni americane a liberarsi dal giogo spagnuolo.

E quali sono questi poemi del gesuiti esiliati? Anzitutto, il Poema Eroico di Diego Giuseppe Abad, vate nato nel messicano Michoacán. È una bella serie di episodi biblici decorati con referenze a Virgilio, ad Ovidio e ad Orazio. La sua edizione integra uscì a Venezia nel 1770. Questo magno volume di 6,000 esametri veniva stampato in fascicoli succesivi, e quel fatto diede occasione ad una di quelle che nel Messico appelliamo "edición pirata" (cioè, copia non autorizzata) contenente metà dei suoi 44 canti, stampati di nascosto nell' ispana città di Cádiz nel 1769. Lo firmava un'anonima "Musa americana", e non il vero autore Diego Giuseppe Abad. L'opera completa è stata tradotta in spagnolo una volta in ciascun secolo. La più recente è del 1974. Ed anche se ne trovano qualcuni canti tradotti all'italiano quando appena erano stati pubblicati.

Poi, è anche assai popolare la Rusticatio Mexicana di Rafaele Landívar. Edita in Modena nel 1780, è una magnifica serie di 6,300 esametri di pittoreschi poemi scalpellati nel Messico e nella Guatemala, nella cui città capitale è nato il poeta. Il suo poema ha goduto di parecchie versioni allo spagnuolo. La più bella in prosa la scrisse Ottaviano Valdés nel 
1940, e poi un'altra in versi endecasilabi la creò Federico Escobedo nel $1924 .^{2}$

Poi, forse il più celebre dei poemi neolatini messicani editi in Italia è stato l'Iliade in versi latini in 15,000 esametri, traduzione firmata nel 1780 dal latinista Francesco Saverio Alegre, nato nel messicano porto di Veracruz intorno al 1700.

Viene dopo, un policromo poema epico dal titolo Guadalupe, creato da un altro inspirato umanista messicano (non gesuita, ma avvocato): Giuseppe de Villerías, il cui manoscritto, di almeno 1,700 versi maggiori, porta la data 1790, ma che é stato stampato solo due secoli dopo nell' Università Nazionale Autonoma del Messico.

\section{Dal secolo XVIII a Francesco Cabrera}

Eppure, dopo quelli bei poemi in gloriosi esametri, per più di due secoli non si pubblicava in America altro che un paio di poemi in quasi cento esametri ciascuno.

Il primo è opera di Modesto Santa Cruz (Brevis descriptio vesperis verni, cioè "Un vespro di primavera", 110 esametri, del 1850). L'altro è la Visio Virgiliana ("Apparizione di Virgilio"; 70 esametri, di 1940) del citato Federico Escobedo.

Ma ecco che, dopo questi modesti neolatinisti del secolo XIX, in metà del secolo Xx lanciasi alle acque neolatine il nuotatore più vigoroso di tutti: il giurista e poeta Francesco Giuseppe Cabrera per dar corso ad una cascata di undici magnifici poemi in esametri la cui corrente, che il poeta annunziava fin dai suoi vent'anni con cento esametri a la Signora di Guadalupe, scaturisce copiosa dalla sua fantasia nel 1988, appena pensionato dalla sua professione di avvocato ai suoi settanta anni, dopo quarantun anni di esercizio.

Questa cascata giunge alla cifra di più di quattro migliaia di esametri neolatini che Cabrera creava felice, in quasi diciasette anni, dal 1988 al 2005.

Dieci di quei poemi sono canti nazionalisti, e l'ultimo è la bella epopea di 700 esametri che esaltano il messaggio mistico e sociale della Regina del Tepeyac.

\footnotetext{
${ }^{2}$ Esiste anche un'altra versione in prosa da Ignazio Loureda, nello stesso 1924.
} 


\section{Tre solide specialità}

Seguiamo prima i passi professionali del nostro geniale poeta. Francesco Cabrera. Nato alla Puebla degli Angeli il 18 marzo 1916. Nella sua gioventù, ha seguito la carriera umanistica coi gesuiti in Ysleta College, e in Nôtre Dame, in Southbend, Indiana, ambedue Università degli Stati Uniti. Ma decisse poi mutare la sua carriera umanistica per quella giuridica nell'Università Nazionale Autonoma del Messico.

Così vedremmo che il nostro Cabrera è un possente latinista, ma è anche un maturo giurista e perfino un acuto critico d'arte. ${ }^{3}$

E così diede dapprima alla stampa la sua tesi di diritto Las libertades del espíritu ("Le libertà dello spirito", nel 1952). Ma poi, pubblicò anche mezza dozzina di lieti libri sulla teoria dell'arte, tali come Pintura, verdad y mito, "Pittura, verità e mito", del 1971.

Eppure fuori dubbio, don Francesco Cabrera occuperà un posto nella storia letteraria come il poeta classicista che, nella bella anzianità, ha creato quasi una dozzina di poemi epici e lirici che ci fanno ricordare $\mathrm{i}$ volumi neolatini di Giovanni Pascoli, l'illustre vate italico di un secolo $\mathrm{fa}$, che fu premiato per dodici anni succesivi nel concorso latino di Amsterdam.

\section{Cabrera canta undici inni neolatini sul Messico}

Orbene, in tutta l'America, nessun'altro latinista di secoli recenti ha saputo creare tutta una costellazione di più di 4,300 esametri come quella che ha dispiegato Francesco Cabrera, la cui produzione neolatina è una cascata inesauribile.

Soltanto si avvicina in fecondità a Cabrera il colombiano Miguel Antonio Caro intorno al 1900.

La professione giuridica di Cabrera lo manteneva sempre attento a personaggi storici non meno che alle città maggiori del suo amato Messico. E, fondendo ambedue professioni, don Francesco crea un ciclo di poemi in classico latino che cantano una vivida successione di città

\footnotetext{
${ }^{3}$ In questo carattere concorreva per parecchi anni al famoso "Círculo del mate paraguayo", di don Ottaviano Valdés.
} 
celebri coi titoli: Tenochtitlan, Angelopolis, Cuernavaca e la leggendaria Tamoanchan.

In quelle stesse città trova Cabrera parecchi eroi e li esalta in poemi che prendono i loro nomi: Quetzalcóatl, Malintzin, Gonzalo Guerrero e Benito Juárez.

Poi, nelle sue amate urbi ascolta Cabrera il canto di Sor Juana Inés de la Cruz nel suo poema Joannae virginis laudes, e sente cantare Amato Nervo nel suo poema Amato Nervo poetae encomium. E finalmente la Madre più amata dei messicani invita Cabrera ad intonare il suo undecimo e culminante poema neolatino maggiore: Laus Guadalupensis ("Exaltación Guadalupana”) nel 1990. ${ }^{4}$

\section{Versi latini memorabili}

Don Francesco Giuseppe è magistrale per ricostruire nel classico latino le memorie perenni del Messico. Così, nel suo canto a Benito Juárez, ricorda che questo usa la definizione del diritto romano nella sua sentenza: "El respeto al derecho ajeno es la paz", e Cabrera la riflette così: $P a$ cis nectit opus qui jura aliena tuetur. ${ }^{5}$ Lo dirò in italiano: "Tesse lavoro di pace chi alieni diritti custodia".

Similmente, quando Cabrera sta lodando Sor Juana Inés de la Cruz, ricorda aver letto in Virgilio l'esametro: Tantae molis erat romanam condere gentem ("Era di tanta importanza fondare la razza romana"). E allora lui fa un esametro simile in onore di Sor Juana: Tantus amor sophiae, tanta est sitis alta sciendi (Joannae, v. 20). In spagnolo: “ ¡Tanto es su amor al saber, tanta es su alta avidez de aprender!". In italiano: "Tanto è il suo amor di sapere, e la sua avidità d'imparare!".

In un parentesi, anch'io assimilo la lezione di Cabrera di scegliere sia dei versi classici latini, sia dei bei canti in lingue moderne per farli risuonare in latino. Allora mi son rammentato della chanconette Aux Champs Elysées. Potremmo cantarlo così, almeno nel suo refrain:

\footnotetext{
${ }^{4}$ L'autore stesso è stato il proprio editore in diverse edizioni: $1^{\mathrm{a}}$ ed., Cuernavaca, 1990; $2^{\text {a }}$ ed., México, 1994; $3^{\text {a }}$ ed., México, 2004, dentro del volume Monumenta Mexicana; $4^{\mathrm{a}}$ ed., México, 2009. Editio recognita, nello stesso volume.

${ }^{5}$ Benedictus Juárez, v. 61.
} 
Aux Champs Elysées (bis)

au soleil, sous la pluie,

à midi ou à minuit.

Il y a tout ce que vous voulez

aux Champs Elysées.

La mia versione latina dice così:

In Campis Elyseis (bis).

Sub sole aut pluvia,

die ac nocte media.

Ibi totum est quod vis

In Elyseis.

Ovvero si può cantare l'Inno all'allegria, di Wilhelm Schiller, che Beethoven intona nella sua IX Sinfonia:

Escucha, hermano, la canción de la alegría, el canto alegre del que espera el nuevo día.

Ven, canta, sueña cantando, vive soñando el nuevo sol

en que los hombres volverán a ser hermanos (bis). ${ }^{6}$

\section{Repertorio}

I. Cabrera canta le sue più amate città del Messico

Adesso seguiamo Francesco Cabrera e faciamo una fermata di uno o due minuti in ciascuno del suoi poemi neolatini.

1. “A Cuernavaca voy, a Cuernavaca!”, cantava l'ispirato Alfonso Reyes quando si allestiva ad interpretare lì nei suoi fini settimana l'omerica Iliade in alessandrini spagnoli.

Così ha potuto cantare anche il nostro Cabrera quando cominciava proclamando il suo inno in bravi esametri a la stessa Cuernavaca, calda

${ }^{6}$ Per parecchi anni abbiamo cantato questa mia versione latina coi miei discepoli: "Exaudi, frater, cantionem exsultantem, / cantum jucundum novum diem expectantem. / Canta, aude, somnia cantans, / vive somnians auram hanc / qua homines cuncti fratres iterum evadent (bis)". 
città della sua residenza di riposo di fine settimana per diecine di anni affine di riscaldare il suo fragile cuore, e poi suo soggiorno abituale appena pensionato, fino al suo tramontato.

Il nome di questo inno? Quauhnahuac. Suburbanae vitae laus. "Cuernavaca, elogio della vita rurale". Si inizia così:

Est locus irriguas inter mitissimus oras...,

Quauhnahuac, piceis sedes contermina silvis (Quauh., vv. 1-3).

Lo traduco in spagnolo:

Hay un dulcísimo sitio entre bien regadas regiones...,

Quauhnáhuac, sede cercana a las selvas boscosas.

E cioè:

C'è un mitissimo luogo, tra ben irrigate regioni...

Quauhnahuac, luogo vicino alle silve boscose.

E similmente continua ad onorare l'amata città di Cuernavaca, con un passaggio che finisce dolcemente così:

Hic "vivo et regno" mansurae pacis alumnus, totus araturo versu studiisque Minervae deditus (vv. 167 ss.).

L'ho tradotto così:

Aquí "vivo y reino", nutrido por una paz duradera, todo dado al verso que crecerá, y de Minerva al estudio.

In italiano lo direi:

Qui “vivo e regno", nutrito da una durevole pace, tutto dato al verso che crescerà e di Minerva allo studio. 
2. Tenochtitlan. Quelli sono radiosi esametri, ma scarsi in numero (soltanto 170 versi), dinnanzi al mezzo migliaio di versi che don Francesco dedica a la città capitale, prima detta Tenochtitlan; oggi, Messico. Cabrera loda la capitale del paese con questi nobili esametri:

Urbs fuit arcanis florens gratissima terris,

Mexicus, uber opum, sceptri decus, horrida bello (Tenoch., vv. 1-2).

Così lo dissi in spagnuolo:

Hubo en arcanas tierras una urbe floreciente y muy grata, México, rica en bienes, noble en reyes, terrible en la guerra.

E in italiano:

C'è stata in arcane terre un'urbe fiorente assai grata, Messico, ricca in averi, illustre in capi, fiera in battaglia.

3. L'Angelopoli. Cabrera consacra poi tutt'un poema a la sua urbe natale: l'Angelopoli (Città degli Angeli), oggi appellata Puebla. Gli diede il titolo Angelopolis: urbis ortus et mirabilia (cioè: "L'Angelopoli. La nascita e le miraviglie della città").

Consta soltanto di 240 esametri, ma qualcuni sono tanto belli come l'esaltazione della sua cattedrale:

Moles magna foro geminas, altissima visu, extollit turres, moles procera colonum (Angelop., vv. 83 s.).

Dico in spagnuolo la mia versione:

En la plaza, magna mole, altísima a la vista, levanta torres gemelas, mole relevante que alzaron colonos.

In italiano direi:

Nella piazza, magna mole, altissima a vedersi, solleva torri gemelle, mole relevante che alzaron coloni. 
4. Tamoanchan. Vicino al suo placido soggiorno di Quauhnáhuac, cioè Cuernavaca, Cabrera contempla il montuoso paradiso che gli sta appresso. Il suo nome è Tepoztlán, ma Cabrera da al riferente inno il nome di Tamoanchan, Elysium (cioè: "la terra dei parenti. Il nostro Elisio, il nostro Paradiso").

In 250 esametri canta don Francesco quel piccolo villaggio con un poema edito nel 1998. Lo inizia nitidamente con un caloroso omaggio al poeta dell'Eneide, nel'esametro VII, 645:

...(liceat modulos iterare Maronis)

"et meministis enim divi et memorare potestis" (Tamoa., vv. 73-75).

Lo interpreto così:

...(séame dado repetir de Marón las palabras)

"pues los dioses lo recordáis y podéis recordárnoslo".

Detto in italiano:

...(mi sia dato ripetere di Maron le parole)

"perche voi divi lo ricordate, capaci a ricordarcelo".

Poi, Cabrera descrive gli olmechi chiavvanzano conquistatori, con esametri come questo:

Nituntur gradibus, montis vi saxa domantes (Tamoa., v. 56).

Detto in spagnuolo:

Suben paso a paso, domando a fuerzas las rocas del monte.

E poi detto in italiano:

Paso a paso ascendon domando a forza le rocche del monte. 
II. Il battaglione degli eroi aztechi

5. Quetzalcoatl. Il secondo gruppo di poemi classici di Cabrera si concentra sugli eroi. Il legendario Quetzalcóatl da nome al quinto magno poema di Cabrera. Lui lo ha scritto nel 1999, nel millenio della nascita di Quetzalcóatl.

Gli olmechi ed i toltechi vedevano questa leggendaria divinità come la incarnazione della nobiltà divina, e gli hanno dedicato superbi monumenti petrei a Teotihuacan, a Cholula ed a Chichén Itzá. Il suo nome significa: "L'ave Quetzal che scintilla come serpente". Cabrera gli consacrò tanti esametri quanti giorni ha un anno naturale: 365 .

La scena della disparizione del eroe è cantata così da don Francesco, coi versi che chiudono il suo poema:

Sic ait, et longo palam super aequora missu jactat; ab attractu ponto subit hospita cymba quam lente scandit. Venti rapuere carinam (Quetzal., vv. 360 ss.).

Lo dico in spagnuolo:

Así dice, y con un gran lance arroja a los mares su manto; a su contacto, del mar surge hospitalaria una barca a la que lento asciende. Los vientos arrastraron la nave.

Lo dico in italiano:

Così dice, e con gran impeto lancia sui mari il suo manto; e al suo contatto sorge ospitaliere una nave su cui ascende lento. La nave rapiscono i venti.

6. Gonzalo Guerrero. Verso la fine del suo ciclo di eroi messicani, il poeta Cabrera dedica un magnifico poema a chi davvero ha creato quella nazionalità.

Il suo titolo? Gonzalo Guerrero, mexicanae Patriae sanguinis ultimus auctor. E cioè: "Gonzalo Guerrero, padre del primo meticcio (mestizo) messicano".

Poema disteso, eh?: quasi 600 versi maggiori, edito nel Messico, 2001. 
Ci sono chi difendano che il primo messicano sia stato figlio di Hernán Cortés e della Malinche.

Ma quanto è vero? Niente affatto.

Gonzalo Guerrero era arrivato nella penisola di Yucatán insieme col padrone di nave Pánfilo di Narváez nel 1511, otto anni prima di Hernán Cortés. Questo Gonzalo diventò amico del cacique (cacico) Nachan, e poi si sposò colla figlia di lui, chiamata Ix-Chel-Can, da cui ebbe tre figli.

Allora, il poeta Francesco Cabrera è uno storico che propugna che $\mathrm{i}$ primi messicani siano stati concepiti dall'amore di una principessa maja e del capitano spagnolo Gonzalo Guerrero. Così lo canta Cabrera:

Imo maja genus formae perculsus honore fovit et uxorem duxit genuitque nepotes, unde nova hispano-majo de sanguine proles.

L'ho tradotto così:

Incluso impulsó la raza maya, inducido al respeto de sus formas, y aquí tomó esposa y engendró descendientes, de do la nueva raza hispano-maya nació de su sangre.

In italiano lo direi:

Anzi, impellì la raza maja, ammirando la grazia delle sue forme, e qui prese sposa e concepì discendenti da dove una nuova razza ispano-maja uscì dal suo sangue.

7. Malintzin, eroina calunniata. Ma non più di un anno dopo, nel 2002, il poeta Cabrera conchiude un nuovo canto maggiore, dall'austero titolo Malintzin.?

Il suo tema è commovente. Malintzin è la donna indiana che offre aiuto al conquistatore Hernán Cortés e diventa la sua interprete (quando non la sua spia) mentre il guerriero va acquistando diversi popoli come alleati contro il re azteco Moctezuma.

\footnotetext{
${ }^{7}$ È frequente capire "malintzin, malinche", come "traditore".
} 
Cabrera ci mostra Malintzin additando a Cortés il passaggio segreto tra le due imponenti montagne nevate Popocatépetl ed Iztaccíhuatl. Ma, alla fine, Malintzin è uccisa da ordini dell'avido e crudele Hernán Cortés.

Qui mostrerò un esametro in cui Cabrera ci ricorda un passaggio celebre dell'Arte poetica di Orazio che dice:

Scimus et hanc veniam petimusque damusque vicissim (Arte, v. 11).

Lo sabemos y esta venia pedimos y a cambio otorgamos.

In italiano:

Lo sappiamo e questa venia chiediamo ed incontro l'offriamo.

Cabrera mostra al cacique offrendo e chiedendo piena lealtà al capitano spagnuolo:

Nos pariter pacemque damus petimusque vicissim (Malin., v. 212).

Lo verto:

Mas por igual te damos la paz y la pedimos a un tiempo.

In italiano:

Noi parimenti ti diamo e ancora ti chiediamo la pace.

8. Juárez, eroe recente. Poi, nel 2005, l'avvocato poeta Francesco Cabrera si unisce a quanti celebrano duecento anni del natale di Benito Juárez. In quell'anno, Cabrera pubblica tutto un solido poema per il "Benemerito". Lì leggiamo esametri come questi:

Alternansque vices regem modo damnat inanem et falsi miserans clemens modo respicit hostem (Juárez, vv. 359 s.).

Verto:

Y alternando opiniones, ora condena al Duque vencido, ora hacia el caído afable, clemente ve al enemigo. 
E cioè in italiano:

E alternando opinioni, ora il Duce vinto condanna, ora soave al caduto, affabile vede il nemico.

III. I poeti canonici del Messico

9. Sor Juana. Finito il ciclo dei suoi poemi per i maggiori eroi del Messico, Cabrera cercava il tema per un altro poema neolatino. Allora ha chiesto agli amici latinisti.

-E se io scrivessi canti classici per i poeti maggiori del mio paese, diciamo: Sor Juana Inés de la Cruz?

Agli amici il tema sembrò eccelente. Felice, don Francesco mise mani all'opera, e cioè penna al foglio. Perfino Cabrera ha cercato qualcuni libri recenti su Sor Juana.

Allora Cabrera inserì nei suoi potenti esametri latini qualcune idee di Sor Juana sviluppate nei celebri sonetti della sua epoca di dama della corte virreinale. Eccone una mostra, un soneto di Sor Juana finisce così:

Por activa y pasiva es mi tormento,

pues padezco en querer y en ser querida.

Nel suo bel latino, Cabrera cantò:

Torqueor infelix, utraque ex parte laboro, uror et optatus torpens mea vota recusat et petor obliquo qui me fastidit amante (Joannae, vv. 118 ss.).

Lo traduco così:

Soy, infeliz, torturada y por ambas partes padezco: me quemo y el querido, insensible, mis deseos rehúsa, y me busca un amante torcido al cual aborrezco.

Direi in italiano:

Son torturata, infelice, che d'ambo le parti io soffro, mi bruccio, ma l'amato, insensible, rifiuta i miei voti, e son cercata da un diverso amante che mi infastidisce. 
10. Nervo il grande. In seguito pensò Cabrera al poeta più affetuoso del Messico: il poeta dell' "amata immobile". Nel seguente anno, il 2003, Cabrera prese il titolo: Amato Nervo poetae encomium. E allora creò 350 esametri per l'amatore di Anne Cécile Daillier, che dopo soli dieci anni diventò "l'amata immobile". Cabrera canta a Anne Cécile, prendendo la ossesione di Amado Nervo:

At nunc Anna, mei crescentis fomes amoris

haeret iners! Verum latis notissima terris

arte mea fiet nulloque tacebitur aevo (Amato, vv. 246 ss.).

La mia versione dice:

¡Pero ahora Anna, acicate de mi amor que se aumenta, yace inerte! Mas se hará famosísima en tierras extensas por arte mía, y en ninguna época va a ser callada.

In italiano direi:

Ma adesso Anna, punzolo del mio amore crescente giace inerte! Ma sarà famosissima in terre distese per arte mia e in tempo nessuno sarà tralasciata.

\section{La Laus Guadalupensis}

11. Francesco Cabrera s'è infiammato nella sua Laudanza guadalupana con uno splendore che non ha uguagliato nei suoi altri dieci grandi poemi neolatini.

Questo poema guadalupano di Cabrera comprende ninetemeno che cinque grandi comparazioni omeriche.

Possiamo esaminare un paio di queste. La prima svolge quell'ave magnifica che è il chetzal, ave che i poeti dicono superiore a tutte le altre avi. Cabrera imagina la Vergine Maria apparsa bella come un chetzal, e gli dedica questi esametri:

Qualis ubi liquidas quetzalis fertur ad auras... eximia comites specie supereminet omnes (Laus, vv. 438-441). 
Lo dico così:

Cual se despliega en las líquidas auras la luz del quetzal... supera a todos sus compañeros en la eximia belleza.

In italiano lo direi:

Qual nelle liquide aure la luce del chetzal si dispiega... supera tutti i compagni nella esimia bellezza.

Un parentesi per la tristissima noctis imago. E si arriva al momento in cui Juan Diego distenderà il suo manto (la indígena "tilma"). Cabrera ricorda qui il più noto esametro di Ovidio:

Cum subit illius tristissima noctis imago.

Lo interpreto:

Cuando me llega de aquella noche la tristísima imagen.

E in italiano:

Quando mi giunge di quella notte il più triste riflesso.

Ma Cabrera la evoca per mostrarci lo stupore dinnanzi alla sacra imagine di Maria:

Numinis apparet formosae matris imago (Laus, v. 663).

Lo decifro così:

La imagen hermosa de la Madre de Dios aparece.

In italiano:

La imagine bella della Madre di Dio si appare.

E come culminazione, Cabrera dispiega uno dei suoi più suggestivi passaggi. È la sua quinta comparazione omerica. Don Francesco com- 
para Juan Diego quando distende il suo manto dinnanzi al vescovo, col pescatore che va colleggendo delle conche nella spiaggia, ma ad un momento si astonisce dinnanzi ad una lucente perla. Così canta Cabrera:

Ut solet interdum piscator littore conchas

vellere cum zephyris positis silet aequoris unda, cui, si forte latens pretiosa repente nitescit gemma, stupet sociosque vocans vox faucibus haeret (Laus, vv. 669 ss.).

In spagnuolo:

Como a veces en la playa el pescador alza unas conchas cuando, calmados los céfiros, la ola del mar se silencia, $\mathrm{y}$, si acaso a él, de repente, una preciosa gema escondida le brilla, se pasma y, llamando a amigos, la voz en su boca se pega.

Ed in italiano:

Come qualvolta alla spiaggia un pescatore abbituato a cogliere delle conche nelle onde, già i zeffiri chetti, se forse a lui subito una preziosa perla nascosta gli brilla, pasmasi e, chiamando amici s'affissa la voce alle sue fauci... ${ }^{8}$

Con questo magnifico canto neolatino, don Francesco Cabrera ha sommato, come repetevamo - repetita juvant-, 4,365 esametri classici.

Questo ciclo che è un monumento di quasi mezzo migliaio di classici esametri corona l'avvocato poeta Francesco Giuseppe Cabrera come il più fecondo poeta classico di America, dopo i geniali gesuiti del secolo XVIII (decimottavo), già prima lodati.

\section{Gli interpreti e gli apologisti}

Tale collana di alta poesia neolatina soltanto è stata tradotta in prosa inglese da William H. Cooper, e in versi esametri spagnuoli da me stes-

${ }^{8}$ Questa scena la ha dipinto il muralista messicano Fernando Leal (Cappella del Cerrito, 1950). Un altro murale nella antica Basilica Guadalupana presenta alti dignitari messicani mostrando la sacra imagine al Papa Benedetto XIII, il quale esclama: Non fecit taliter omni nationi ("Non ha fatto una cosa eguale a nessun'altra nazione"). 
so. Cooper accordò coll'autore denominare tutta la serie: Monumenta Mexicana, cioè Mexican Heritage. Direi anche: "La magna eredità messicana".

Ed è vero. Questa imponente collana di poemi epici lirici sono altretante gemme della storia del Messico, ricamate da potenti esametri latini.

Poi, l'illustre poeta Francesco Cabrera ha ascoltato omaggi dalla dottoressa Nancy Llewellyn, e poi dai dottori Albert Baca, Craig Kallendorf, Andrew Laird, e dal avvocato messicano Alfredo Borboa. E Cabrera ha ricevuto encomi perfino dal Giappone.

Concludendo, riferirò che, nel doppio centenario dell'indipendenza del Messico, licenziato Cabrera era stato invitato a comporre un altro inno epico allusivo. Lui l'intitolò Libertatis Gesta ("Le gesta della libertà").

Don Francesco compose i primi 45 esametri di quel proiettato poema, ma dopo quelli ha finalmente desistito. Esausto, non si sentiva più capace di creare ancora più esametri latini. Ai suoi 94 anni aveva già scritto abbastanza.

Don Francesco continuò a vivere fino ai 99 anni. Non vide più la sua Laus Guadalupensis, ${ }^{9}$ oggi in processo di edizione, coi suoi 700 esametri. Don Francesco Giuseppe Cabrera è già immortale.

Salve, Francisce, poeta perennis!

\section{BIBLIOGRAFIA}

CABrera, Francisco José, Las libertades del espíritu, tesis de licenciatura en derecho, México, Universidad Nacional Autónoma de México, 1960.

CABrera, Francisco José, Italia. Guía de contemplativos (Recuerdos de viaje) México, 1967.

CABrera, Francisco José, Tres ciudades, tres cantos neolatinos: Mexicus Tenochtitlan, Angelopolis, Quauhnahuac, estudio y versión rítmica Tarsicio Herrera Zapién, México, Universidad Nacional Autónoma de México, 2004.

CABrera, Francisco José, Sor Juana y Nervo en latinidad lírica, estudio, versión rítmica y notas Tarsicio Herrera Zapién, Universidad Nacional Autónoma de México, 2007.

\footnotetext{
${ }^{9}$ La versione in spagnolo si chiamerà Exaltación Guadalupana.
} 
CABrera, Francisco José, Tribunus ille Benedictus Juárez (Benito Juárez, tribuno de bronce), estudio, versión rítmica y notas Tarsicio Herrera Zapién, Universidad Nacional Autónoma de México, 2008.

CABrera, Francisco José, Monumenta Mexicana. Los once poemas épico-líricos, con versión al inglés William C. Cooper, México, 2004 y 2009.

CABrera, Francisco José, Gonzalo Guerrero y Malintzin. Dos poemas neolatinos, introd., versión rítmica y notas Tarsicio Herrera Zapién, México, Universidad Nacional Autónoma de México, 2010.

CABrera, Francisco José, Quetzalcóatl y el paraíso de Tamoanchan. Dos poemas neolatinos, estudio, versión rítmica y notas Tarsicio Herrera Zapién, México, Universidad Nacional Autónoma de México, 2012.

CABrera, Francisco José, Exaltación Guadalupana. Laus Guadalupensis. Tradición y recepción clásico-moderna, estudio, versión rítmica y notas Tarsicio Herrera Zapién, México, Universidad Nacional Autónoma de México, en prensa. 